\title{
A CLASS OF NONLINEAR STURM-LIOUVILLE PROBLEMS WITH INFINITELY MANY SOLUTIONS
}

\author{
RENATE SCHAAF AND KLAUS SCHMITT
}

ABStraCt. This paper is concerned with the existence of solutions of nonlinear Sturm-Liouville problems whose linear part is at resonance. It is shown that such problems may have infinitely many solutions if the nonlinear perturbations are periodic.

1. Introduction. Consider the linear inhomogeneous problem

$$
\left\{\begin{array}{l}
u^{\prime \prime}+u=h(x), \quad 0 \leq x \leq \pi, \\
u(0)=0=u(\pi) .
\end{array}\right.
$$

Then, of course, (1) has infinitely many solutions whenever

$$
\int_{0}^{\pi} h(x) \sin x d x=0
$$

The question then arises whether solutions will exist for nonlinear perturbations of (1), i.e., boundary value problems of the form

$$
\left\{\begin{array}{l}
u^{\prime \prime}+u+g(u)=h(x), \quad 0 \leq x \leq \pi, \\
u(0)=0=u(\pi) .
\end{array}\right.
$$

One of the first papers to treat problems with linear part at resonance is the paper [LL] by Landesman and Lazer; the results and methods developed in this paper have been extended and refined in many different directions during recent years. Most recently Ward [W] showed that if $h$ satisfies (2) and $g$ is a nontrivial periodic nonlinearity of zero mean then in fact (3) is solvable. This result was extended to the case of elliptic partial differential equations and the case of higher eigenvalues in $[\mathbf{S}, \mathbf{L S}]$. In this paper we shall show that as a consequence of our main result Ward's problem (2) will have, in fact, infinitely many positive and infinitely many negative solutions. This we demonstrate by using a somewhat different approach, namely techniques from bifurcation theory. We embed (3) into a one-parameter problem

$$
\left\{\begin{array}{l}
u^{\prime \prime}+\lambda u+g(u)=h(x), \quad 0 \leq x \leq \pi, \\
u(0)=0=u(\pi)
\end{array}\right.
$$

and show that (4) has a continuum of solutions $(\lambda, u)$ which bifurcates from infinity at $\lambda=1$. We then show that this continuum must in fact cross the $\lambda=1$ level

Received by the editors October 6, 1986 and, in revised form, March 6, 1987.

1980 Mathematics Subject Classification (1985 Revision). Primary 34B15; Secondary 47H15, $58 \mathrm{E} 07$.

Research supported by the Deutsche Forschungsgemeinschaft and an NSF grant. 
infinitely often. The reason that the latter is true is that, whenever $g$ is periodic and of zero mean, the integral

$$
\int_{0}^{\pi} g(\alpha \sin x) \sin x d x
$$

is an oscillatory function of $\alpha$ near $\alpha= \pm \infty$. A similar result (in the case of semilinear elliptic problems) is unavailable to us at this time; we hence restrict ourselves to the case of nonlinear Sturm-Liouville problems.

This paper is organized in the following way. $\S 2$ contains the statement of our main result, $\S 3$ is devoted to an abstract result about bifurcation from infinity, whose essential features follow from results in $[\mathbf{R}, \mathbf{P S}]$, and in $\S 4$ we use these ideas together with some other auxiliary results to prove our main theorem.

This work was accomplished at SFB 123 of the University of Heidelberg during a recent visit of the second author to that institution. Both authors acknowledge some valuable insight provided by Stefan Luckhaus.

2. The main result. We shall consider nonlinear Sturm-Liouville problems of the following type:

$$
\left\{\begin{array}{l}
u^{\prime \prime}+\lambda_{0} r u+p g(u)=h, \quad 0 \leq x \leq 1, \\
a_{0} u(0)-b_{0} u^{\prime}(0)=0=a_{1} u(1)+b_{1} u^{\prime}(1) .
\end{array}\right.
$$

The coefficients in problem (6) are to satisfy the requirements

$$
a_{i}, b_{i} \geq 0, \quad i=0,1, \quad\left(a_{0}+b_{0}\right)\left(a_{1}+b_{1}\right)>0, \quad a_{0}+a_{1}>0,
$$

$\lambda_{0}>0$ is the principal eigenvalue with eigenfunction $\phi$ of the eigenvalue problem,

$$
\begin{gathered}
\left\{\begin{array}{l}
u^{\prime \prime}+\lambda r u=0, \quad 0 \leq x \leq 1, \\
a_{0} u(0)-b_{0} u^{\prime}(0)=0=a_{1} u(1)+b_{1} u^{\prime}(1)
\end{array}\right. \\
\int_{0}^{1} h(x) \phi(x) d x=0
\end{gathered}
$$

$$
\begin{gathered}
r \in C^{1}\left([0,1], \mathbf{R}^{+}\right), p \in C^{2}\left([0,1], \mathbf{R}^{+}\right) \text {with either } p \equiv \text { constant or } \\
\qquad p^{\prime} \phi \phi^{\prime}+p\left(\phi^{\prime}\right)^{2}+\lambda_{0} p r \phi^{2}>0 \text { on }[0,1] .
\end{gathered}
$$

Concerning the nonlinear term $g$ in our problem we require

$$
g: \mathbf{R}^{+} \rightarrow \mathbf{R} \text { is continuous with } \frac{g(s)}{s} \rightarrow 0 \quad \text { as } s \rightarrow \infty
$$

There exist sequences $\left\{s_{n}\right\}$ and $\left\{t_{n}\right\}$ with $s_{n} \rightarrow \infty, t_{n} \rightarrow \infty$ and

$$
\int_{s}^{s_{n}} g(t) d t \geq 0 \quad \text { for all } s<s_{n}, \quad \int_{s}^{t_{n}} g(t) d t \leq 0 \text { for all } s<t_{n} .
$$

We note that if $g$ is a continuous periodic function with period $T$ having mean value $0, \int_{0}^{T} g(t) d t=0$, then $g$ will satisfy requirements (12) and (13). In this case the 
primitive $G(s)=\int_{0}^{s} g(t) d t$ is a periodic function of period $T, G(0)=G(T)=0$. We let $s_{1}$ and $t_{1}$ be such that

$$
G\left(s_{1}\right)=\max _{[0, T]} G(s), \quad G\left(t_{1}\right)=\min _{[0, T]} G(s) .
$$

Then $G\left(s_{1}\right) \geq 0 \geq G\left(t_{1}\right)$ and for $s_{n}=s_{1}+(n-1) T, t_{n}=t_{1}+(n-1) T$ we have

$$
\int_{s}^{s_{n}} g(t) d t=G\left(s_{1}\right)-G(s) \geq 0, \quad \int_{s}^{t_{n}} g(t) d t=G\left(t_{1}\right)-G(s) \leq 0 .
$$

Using periodic functions $g$ one may easily construct nonperiodic functions satisfying (13), viz. $g(s)=s^{q-1} \sin s^{q}, 0<q<2$, is such a function.

We are now in a position to state our main result.

THEOREM 1. Assume that the requirements (7)-(13) are satisfied. Then the nonlinear boundary value problem (6) has an infinite sequence of positive solutions $\left\{u_{n}\right\} \subseteq C^{2}\left([0,1], \mathbf{R}^{+}\right)$with the property

$$
\max u_{n} \rightarrow \infty, \quad \frac{u_{n}}{\max u_{n}} \rightarrow \phi \quad \text { as } n \rightarrow \infty .
$$

3. On bifurcation from infinity. In this section we shall discuss a theorem about continua of solutions of nonlinear equations which bifurcate from infinity. The theorem is similar in nature to the results in [R, PS]; in fact it follows from these results. However, for the sake of completeness we shall give a formulation here which is suitable for our purpose.

Let $\mathbf{X}$ be a real Banach space with norm $\|\cdot\|, \Lambda \subset \mathbf{R}$ be an open interval, and $L: \Lambda \rightarrow \mathbf{L}(\mathbf{X}, \mathbf{X})$ be a family of compact linear operators which is differentiable on $\Lambda$.

We consider the equation

$$
u=L(\lambda) u+H(\lambda, u)
$$

where $H: \Lambda \times \mathbf{X} \rightarrow \mathbf{X}$ is completely continuous and satisfies

$$
\frac{H(\lambda, u)}{\|u\|} \rightarrow 0 \text { as }\|u\| \rightarrow \infty, \text { uniformly on compact subsets of } \Lambda \text {. }
$$

THEOREM 2. Assume there exists $\lambda_{0} \in \Lambda$ such that

$$
\begin{gathered}
\operatorname{ker}\left(\mathrm{id}-L\left(\lambda_{0}\right)\right)=\operatorname{span} \phi, \quad\|\phi\|=1, \\
L^{\prime}\left(\lambda_{0}\right) \phi \notin \operatorname{range}\left(\mathrm{id}-L\left(\lambda_{0}\right)\right) .
\end{gathered}
$$

Then if $\mathbf{K} \subset \mathbf{X}$ is an open cone with $\phi \in \mathbf{K}$ and $H$ satisfies (16) we have:

There exists a continuum $C \subset \Lambda \times \mathbf{K}$ of solutions of (15) with the property that for all $\varepsilon, 0<\varepsilon \leq \varepsilon_{0}$, there exists a subcontinuum $C_{\varepsilon}$ of $C$,

$$
C_{\varepsilon} \subset U_{\varepsilon}\left(\lambda_{0}, \infty\right):=\left\{(u, \lambda):\|u\|>1 / \varepsilon,\left|\lambda-\lambda_{0}\right|<\varepsilon\right\},
$$

and $C_{\varepsilon}$ connects $\left(\lambda_{0}, \infty\right)$ to $\partial U_{\varepsilon}\left(\lambda_{0}, \infty\right)$. Moreover, if $\left(\lambda_{n}, u_{n}\right) \in C \cap U_{\varepsilon_{0}}\left(\lambda_{0}, \infty\right)$ with $\left\|u_{n}\right\| \rightarrow \infty$, then

$$
\lambda_{n} \rightarrow \lambda_{0} \quad \text { and } \quad \frac{u_{n}}{\left\|u_{n}\right\|} \rightarrow \phi, \quad n \rightarrow \infty .
$$


Furthermore, $C$ has the following additional global properties:

If $C$ does not have a limit point in $\partial(\Lambda \times \mathbf{K})$, then either

(i) $C$ is unbounded in $\lambda$, or

(ii) $C$ has a limit point $\left(\lambda_{1}, \infty\right)$ with $\lambda_{0} \neq \lambda_{1} \in \Lambda$ and $\operatorname{ker}\left(\mathrm{id}-L\left(\lambda_{1}\right)\right) \neq\{0\}$.

REMARK. In many bifurcation problems it follows that the bifurcating branches may, at least locally, be parametrized by $\lambda$. This is not necessarily the case in the above theorem (and in fact it will not be true in our particular situation) as one may see, using the approach of Rabinowitz [R], and inverting the problem on the unit ball and treating an equivalent problem of bifurcation from the trivial solution. The resulting nonlinear operator, while differentiable at 0 , will not be of class $C^{1}$ in a neighborhood of 0 .

4. The bifurcation problem. In order to prove Theorem 1 we embed (6) into the one-parameter problem

$$
\left\{\begin{array}{l}
u^{\prime \prime}+\lambda r u+p g(u)=h, \quad 0 \leq x \leq 1, \\
a_{0} u(0)-b_{0} u^{\prime}(0)=a_{1} u(1)+b_{1} u^{\prime}(1) .
\end{array}\right.
$$

Since $g$ was only defined on $[0, \infty)$ we extend $g$ to $(-\infty, \infty)$ by setting $g(s):=g(0)$ for $s \leq 0$, The way $g$ is extended for negative values of $s$ is, of course, unimportant since only positive solutions are of interest here. As Banach spaces we shall use

$$
\mathbf{X}:=C^{0}([0,1], \mathbf{R})
$$

with norm $\|\cdot\|$ and

$$
\mathbf{Y}=\left\{u \in C^{2}([0,1], \mathbf{R}): a_{0} u(0)-b_{0} u^{\prime}(0)=0=a_{1} u(1)+b_{1} u^{\prime}(1)\right\}
$$

with norm $\|\cdot\|_{2}$.

Using Green's functions, or operator theoretic methods, we may convert (20) into a fixed point problem

$$
u=-\lambda i \circ \operatorname{Tr} u-i \circ T(p g(u)-h),
$$

where $i: \mathbf{Y} \rightarrow \mathbf{X}$ is the compact injection of $\mathbf{Y}$ into $\mathbf{X}$ and $T$ is the bounded inverse of the differential operator $A: \mathbf{Y} \rightarrow \mathbf{X}, A u=u^{\prime \prime}$.

Letting $L(\lambda)=-\lambda i \circ T r$ and $H(\lambda, \cdot)=-i \circ T(p g(\cdot)-h)$ we easily see that (16)-(18) are satisfied, where $\phi$ is given as in (10). We choose $\mathbf{K}$ as

$$
\mathbf{K}:=\left\{u \in \mathbf{X}: \int_{0}^{1} u \phi d x>0\right\} .
$$

We see that all assumptions of Theorem 2 are satisfied and we hence obtain a continuum $C \subset \mathbf{R} \times \mathbf{K}$ of solutions of (21) with the properties listed in Theorem 2. We note that $C$ may also be regarded as a continuum in $\mathbf{R} \times \mathbf{Y}$. Moreover, if $\left(\lambda_{n}, u_{n}\right) \in C \cap U_{\varepsilon_{0}}\left(\lambda_{0}, \infty\right)$ with $\left\|u_{n}\right\| \rightarrow \infty$, then $\lambda_{n} \rightarrow \lambda_{0}$ and $u_{n} /\left\|u_{n}\right\| \rightarrow \phi$ as $n \rightarrow \infty$. The last limit will also hold with respect to the topology of $\mathbf{Y}$. Hence Theorem 2 will also hold if $\mathbf{K}$ is any open cone in $\mathbf{Y}$ with $\phi \in \mathbf{K}$. We therefore choose

$$
\begin{aligned}
\mathbf{K}:=\left\{u \in \mathbf{Y}: u(x)>0, x \in(0,1), u(0)>0 \text { or } u^{\prime}(0)\right. & >0, \\
u(1) & \left.>0 \text { or } u^{\prime}(1)<0\right\} .
\end{aligned}
$$

It also follows from properties of linear differential equations that alternative (ii) of Theorem 2 cannot occur in our problem.

We summarize these considerations in the following corollary. 
COROLlaRY 3 . Let $\mathbf{K}$ be given by (22). Then there exists a connected set $C \subset \mathbf{R} \times \mathbf{K}$ of solutions of (20) having the following properties:

(i) For all $0<\varepsilon \leq \varepsilon_{0}$ there exists a subcontinuum $C_{\varepsilon} \subset U_{\varepsilon}\left(\lambda_{0}, \infty\right)$ of $C$ which approaches $\left(\lambda_{0}, \infty\right)$ and $\partial U_{\varepsilon_{0}}\left(\lambda_{0}, \infty\right)$ in the $\mathbf{R} \times \mathbf{X}$ topology.

(ii) If $\left(\lambda_{n}, u_{n}\right) \in U_{\varepsilon_{0}}\left(\lambda_{0}, \infty\right)$ with $\left\|u_{n}\right\| \rightarrow \infty$ as $n \rightarrow \infty$, then $\left\|u_{n} /\right\| u_{n}\|-\phi\|_{2} \rightarrow$ 0 and $\lambda_{n} \rightarrow \lambda_{0}$ as $n \rightarrow \infty$.

(iii) If $C$ does not have a limit point (with respect to $\mathbf{R} \times \mathbf{Y}$ ) in $\mathbf{R} \times \partial \mathbf{K}$, then $C$ is unbounded with respect to $\lambda$.

Using (iii) we get the following corollary.

COROLlaRY 4. Let $g(0)>\|h / p\|$. Then there exists a continuum $C \subset \mathbf{R} \times \mathbf{K}$ of solutions of (20) with the properties stated in Corollary 3 and

$$
C \subset \mathbf{R} \times \mathbf{K}, \quad C \text { is unbounded in } \lambda .
$$

PrOOF. It suffices to show that $C$ may not have a limit point in $\mathbf{R} \times \partial \mathbf{K}$. If there exists $(\bar{\lambda}, \bar{u}) \in \mathbf{R} \times \partial \mathbf{K} \cap \bar{C}$ then it is a solution of (2.6) and there exists $x_{0} \in[0,1]$ with $\bar{u}\left(x_{0}\right)=\bar{u}^{\prime}\left(x_{0}\right)=0$ and $\bar{u}^{\prime \prime}\left(x_{0}\right) \geq 0$. Thus

$$
0 \leq \bar{u}^{\prime \prime}\left(x_{0}\right)=-p\left(x_{0}\right) g(0)+h\left(x_{0}\right)<-\left|h\left(x_{0}\right)\right|+h\left(x_{0}\right) \leq 0,
$$

a contradiction.

5. Proof of Theorem 1 and remarks. We shall now employ what has been established in the previous section and show that the continua discussed there in fact cross the $\lambda=\lambda_{0}$ level infinitely often hence giving rise to the existence of infinitely many positive solutions of (6). For given $\varepsilon>0$, let $C_{\varepsilon}$ be the continuum of solutions of (20) obtained in Corollary 3. Taking the $L^{2}$-inner product of $(20)$ with $\phi$ we obtain (since (11) holds)

$$
\left(\lambda_{0}-\lambda\right) \int_{0}^{1} r u \phi d x=\int_{0}^{1} p g(u) \phi d x .
$$

Hence for any solution in $C_{\varepsilon}$ the sign of $\left(\lambda_{0}-\lambda\right)$ is determined by the right-hand side of (24). If $\varepsilon$ is small enough then $\|u /\| u\|-\phi\|_{2}$ is small for all $(\lambda, u) \in C_{\varepsilon}$. One easily sees that $\phi^{\prime}$ has exactly one zero $x_{0}$ in $[0,1]$ and

$$
\phi\left(x_{0}\right)=\max \phi=1, \quad \phi^{\prime \prime}\left(x_{0}\right)<0 .
$$

Hence for such small $\varepsilon$ any zero $x_{1}$ of $u^{\prime}$, where $(\lambda, u) \in C_{\varepsilon}$, must be close to $x_{0}$, and $u^{\prime \prime}\left(x_{1}\right)<0$. Then any $u$, with $(\lambda, u) \in C_{\varepsilon}$, will have exactly one critical point $x_{1}$ and $u\left(x_{1}\right)=\max u=\|u\|$. Also $u^{\prime}(x)>0$ on $\left[0, x_{1}\right)$ and $u^{\prime}(x)<0$ on $\left(x_{1}, 1\right]$. Let $G(s)=\int_{0}^{s} g(t) d t$. Then for all $(\lambda, u) \in C_{\varepsilon}$

$$
\begin{aligned}
\int_{0}^{1} p(x) g(u(x)) \phi(x) d x \\
=\int_{0}^{1} \frac{d}{d x}\left[G(u(x))-G\left(u\left(x_{1}\right)\right)\right] \frac{p(x) \phi(x)}{u^{\prime}(x)} d x \\
=\frac{p(1) \phi(1)}{u^{\prime}(1)}[G(u(1))-G(\|u\|)]-\frac{p(0) \phi(0)}{u^{\prime}(0)}[G(u(0))-G(\|u\|)] \\
\quad+\int_{0}^{1}(G(\|u\|)-G(u(x))) \frac{p^{\prime} \phi u^{\prime}+p \phi^{\prime} u^{\prime}-p \phi u^{\prime \prime}}{\left(u^{\prime}\right)^{2}} d x .
\end{aligned}
$$


Integration by parts is allowed here since the function

$$
\frac{G\left(u\left(x_{1}\right)\right)-G(u(x))}{\left(u^{\prime}(x)\right)^{2}}
$$

has a finite limit as $x \rightarrow x_{1}$. For the same reason if either $u^{\prime}(0)=0$ or $u^{\prime}(1)=0$, then the corresponding boundary term vanishes. We note that, since both $u$ and $\phi$ satisfy the boundary conditions we have that $\phi(1) u^{\prime}(1) \leq 0$ and $\phi(0) u^{\prime}(0) \geq 0$. Hence, in order to determine the sign of (24) it will be important to study the sign of

$$
p^{\prime} \phi u^{\prime}+p \phi^{\prime} u^{\prime}-p \phi u^{\prime \prime} .
$$

Since for $\varepsilon>0$ small $u /\|u\|$ is close to $\phi$ in the $C^{2}$ (i.e., $\mathbf{Y}$ ) norm, it suffices to study the sign of

$$
f:=p^{\prime} \phi \phi^{\prime}+p\left(\phi^{\prime}\right)^{2}-p \phi \phi^{\prime \prime} .
$$

If $p \equiv$ const, then $f(x)>0$ on $[0,1]$ since $\phi$ cannot have any double zeros. Otherwise (11) implies that $f$ is positive (some conditions on $p$ and $r$ which imply that (11) holds will be given below).

Now $C_{\varepsilon}$ is a connected set which contains solutions $(\lambda, u)$ with $\|u\|=c$ for any $c$ large enough. Thus taking the sequences $\left\{s_{n}\right\}$ and $\left\{t_{n}\right\}$ from condition (13) we find that for any $n$, sufficiently large, there exist $\left(\lambda_{n}, u_{n}\right)$ and $\left(\lambda_{n}^{\prime}, v_{n}\right) \in C_{\varepsilon}$ such that

$$
\left\|u_{n}\right\|=s_{n}, \quad\left\|v_{n}\right\|=t_{n},
$$

hence

$$
G\left(\left\|u_{n}\right\|\right)-G\left(u_{n}(x)\right) \geq 0, \quad G\left(\left\|v_{n}\right\|\right)-G\left(v_{n}(x)\right) \leq 0
$$

and therefore, using (24), we get

$$
\int_{0}^{1} p(x) g\left(u_{n}(x)\right) \phi(x) d x \geq 0 \geq \int_{0}^{1} p(x) g\left(v_{n}(x)\right) \phi(x) d x .
$$

Interlacing these sequences appropriately we find therefore that the continuum $C_{\varepsilon}$ must cross the $\lambda=\lambda_{0}$ level infinitely often, hence proving our result.

One of the crucial assumptions of Theorem 1 is that the sign of the expression $f$ given in (26) is positive. As remarked above, this certainly is the case if $p \equiv$ const as is assumed in [W]. In the following lemma we give another situation which allows one to deduce the positivity of $f$.

LEMMA 5. Let the coefficient functions $p$ and $r$ satisfy

$$
\begin{aligned}
& 2\left(p^{\prime}\right)^{2}-p p^{\prime \prime}>0 \quad \text { on }[0,1], \\
& p^{\prime} r^{\prime}-r p^{\prime \prime} \geq 0 \quad \text { on }[0,1] .
\end{aligned}
$$

Then $f$, given by $(26)$, is positive on $[0,1]$.

PROOF. It follows from (27) and the positivity of $p$ that there exists $x_{2} \in[0,1]$ such that $p^{\prime}>0$ on $\left[0, x_{2}\right)$ and $p^{\prime}<0$ on $\left(x_{2}, 1\right]$. Furthermore as observed earlier $\phi^{\prime}$ satisfies $\phi^{\prime}>0$ on $\left[0, x_{0}\right)$ and $\phi^{\prime}<0$ on $\left(x_{0}, 1\right]$. Hence, since

$$
f=p^{\prime} \phi \phi^{\prime}+p\left(\phi^{\prime}\right)^{2}+\lambda_{0} r p \phi^{2},
$$


it is the case that

$$
f>0 \text { on }[0, a] \text { and }[b, 1],
$$

where $a=\min \left\{x_{0}, x_{2}\right\}, b=\max \left\{x_{0}, x_{2}\right\}$, and where we again have used that $\phi$ has do double zeros. Thus if $f$ is not positive on the whole interval $[0,1]$ it must have a nonpositive minimum in $(a, b)$.

We compute

$$
f^{\prime}=p^{\prime \prime} \phi \phi^{\prime}+2 p^{\prime}\left(\phi^{\prime}\right)^{2}+\lambda_{0} p r^{\prime} \phi^{2}
$$

and hence

$$
p^{\prime} f^{\prime}=p^{\prime \prime} f+\left(2\left(p^{\prime}\right)^{2}-p p^{\prime \prime}\right)\left(\phi^{\prime}\right)^{2}+\lambda_{0} p\left(p^{\prime} r^{\prime}-r p^{\prime \prime}\right) \phi^{2},
$$

from which by (27) and (28) it follows that

$$
p^{\prime} f^{\prime} \geq p^{\prime \prime} f \quad \text { on }[0,1], \quad\left(p^{\prime} f^{\prime}\right)(x)>\left(p^{\prime \prime} f\right)(x), \quad x \neq x_{0} .
$$

If $\bar{x} \in(a, b)$ is the first zero of $f$ then $\bar{x} \neq x_{0}$, and it follows from (29) that $p^{\prime}(\bar{x})<0$, $f^{\prime}(\bar{x})<0$. Thus $a=x_{2}$, and again by (29) $f$ cannot have a second zero on $(a, b)$, a contradiction. Hence $f>0$ on $[0,1]$.

REMARK. There is a large class of functions $p$ satisfying (27) since the condition follows from $(\ln p)^{\prime \prime} \leq 0$. Condition $(28)$ is not so nice, but it holds, for example, if $(\ln p)^{\prime \prime} \leq 0, r=a p$, and $a^{\prime} p^{\prime} \geq 0$, or for $r \equiv$ const and $p^{\prime \prime} \leq 0$.

REMARK. It is clear from the proof of Theorem 1 how one may deduce the existence of infinitely many negative solutions of Ward's problem [W].

\section{REFERENCES}

[CR] M. Crandall and P. R. Rabinowitz, Bifurcation from simple eigenvalues, J. Funct. Anal. 8 (1973), 161-180.

[LL] E. M. Landesman and A. C. Lazer, Nonlinear perturbations of linear elliptic boundary value problems at resonance, J. Math. Mech. 19 (1970), 609-623.

[LS] D. Lupo and S. Solimini, A note on a resonance problem, preprint, ISAS, Trieste, 1985.

[PS] H. O. Peitgen and K. Schmitt, Global analysis of two-parameter elliptic eigenvalue problems, Trans. Amer. Math. Soc. 283 (1984), 57-95.

[R] P. H. Rabinowitz, On bifurcation from infinity, J. Differential Equations 14 (1983), 462-475.

[S] S. Solimini, On the solvability of some elliptic partial differential equations with linear part at resonance, preprint, ISAS, Trieste, 1984.

[W] J. R. Ward, A boundary value problem with a periodic nonlinearity, Nonlinear Anal. 10 (1986), 207-213.

SONDERFORSCHUNGSBEREICH 123, UNIVERSITÄT HEIDELBERG, IM NEUENHEIMER FELD 294, D-6900 HeIDElBerg, West Germany

Department of Mathematics, University of Utah, Salt Lake City, Utah 84112 